\title{
Search for high energy neutrinos from bright GRBs with ANTARES
}

\author{
M. Sanguineti ${ }^{1,2, a}$, S. Celli $i^{3,4}$, and D. Turpin ${ }^{5}$ on behalf of the ANTARES collaboration \\ ${ }^{1}$ Dipartimento di Fisica dell'Università, Via Dodecaneso 33, 16146, Genova, Italy \\ ${ }^{2}$ INFN-Sezione di Genova, Via Dodecaneso 33, 16146, Genova, Italy \\ ${ }^{3}$ Gran Sasso Science Institute, Viale Francesco Crispi 7, 00167, L'Aquila, Italy \\ ${ }^{4}$ INFN-Sezione di Roma, P.le Aldo Moro 2, 00185, Roma, Italy \\ ${ }^{5}$ Aix Marseille Université, CNRS/IN2P3, CPPM UMR 7346, 13288, Marseille, France
}

\begin{abstract}
Gamma-ray bursts are a possible site of hadronic acceleration, thus neutrinos are expected in correspondence of a GRB event. The brightest GRB observed between 2008 and 2013 (GRB080916C, GRB110918A, GRB130427A and GRB130505A) have been investigated using the data of the ANTARES high energy neutrino telescope. In this paper two of most promising models of the GRB neutrino emission will be studied: the internal shock model and the photospheric model. No muons have been measured in space and time correlation with the selected GRBs and upper limits at 90\% C.L. on the expected neutrino fluxes have been derived. This measure allows also setting constraints on some parameters used in the modeling of the neutrino flux: the bulk Lorentz factor of the jet $\Gamma$ and the baryon loading $f_{p}$.
\end{abstract}

\section{Introduction}

The ANTARES detector (Astronomy with a Neutrino Telescope and Abyss environmental RESearch) is the largest neutrino telescope currently in operation in the Northern Hemisphere and the first operating in sea water. It is a three dimensional array of photo-multiplier tubes (PMTs), in which neutrinos are detected through the Cherenkov radiation induced by ultra-relativistic particles created in a neutrino interaction. The main goal of the ANTARES telescope is the detection of high energy cosmic neutrinos and in particular the identification of point-like sources.

In particular GRBs are one of most interesting types of celestial objects, they represent the most powerful outburst of energy in the Universe since the Big Bang itself. Gamma ray bursts are intense flashes of gamma rays, whose duration can vary from a fraction of a second up to a few minutes [1] [2]. Several gamma ray burst models have been proposed in the last few years, the most promising of which are the internal shock [1] and photospheric models [3] (see Sect. 2). Both scenarios predict a neutrino flux in correspondence of a GRB event. ANTARES ([4] and [5]) and IceCube ([6] and [7]) have already performed several research of a neutrino flux from GRBs, but they did not succeed in the identification of a significant excess of events over the expected background.

ae-mail: matteo.sanguineti@ge.infn.it 
In Sec. 2 the two neutrino emission mechanism (internal shock and photospheric) are introduced and in Sec. 3 the analysis approaches will be described. Finally in Sec. 4 the derived upper limits on the neutrino flux and the constraints on the GRBs parameters will be presented (Sec. 5).

\section{The internal shock and photospheric models}

Both internal shock and photospheric models assume that the gamma ray emission is due to a relativistic jet of particle ejected by an inner engine, but the location of the interaction is different. In the case of the internal shock model the gamma rays are produced by the interaction of different shock waves inside the jet, on the other hand in the photospheric scenario the interaction takes place in the initial part of the expansion of the jet, when it is still opaque to photons.

The predicted neutrino energetic range is completely different in the two scenarios. In the case of the internal shock model neutrino are expected to be above $100 \mathrm{TeV}$ [1], while in the photosphetic picture a low energy component is predicted [3]. For the internal shock model the GRB neutrino spectra have been computed thanks to the numerical code 'NeuCosmA' [8] and in the case of the photospheric scenario the analytical approach in [3] have been used. Both predictions rely on the measured parameters of the $\gamma$-ray emission light curve and spectrum, and assume as bulk Lorentz factor of the jet $\Gamma=316$ and as baryon loading $f_{p}=10$.

\section{GRB selection and analysis}

The brightest GRBs provide more probability of a neutrino flux discovery, because the per-burst neutrino fluence is directly scaled to the $\gamma$-ray fluence. The GRBs included in this analysis have a $\gamma$-ray fluence $F_{\gamma}$ larger than $1 \times 10^{-4} \mathrm{erg} \mathrm{cm}^{-2}$. The selected GRBs were also below the horizon at the trigger time and information on the redshift of the bursts are available. Four bright GRBs fulfil all the requirements: GRB080916C, GRB110918A, GRB130427A and GRB130505A.

The data acquisition system of the ANTARES neutrino telescope is based on the unique "all-datato-shore" concept: all photon signals recorded by the detector are transported to the shore station where filtering is performed. The filtering algorithms are also operating during GRB events, but in this case also raw data are saved on disks for a couple of minutes, so that data cover the majority of the burst duration. Because of the different energy ranges of the searches, in the internal shock model case the filtered data are used [9], while for the photospheric model study raw data are exploited. In both analysis the aperture of the search cone around the burst is set to $10^{\circ}$, while the search time window in the internal shock analysis is fixed to be equal to each burst duration with a symmetric extension of 2 seconds, while in the photospheric model case it depends on the raw data buffer duration. Since for GRB080916C and GRB110918A raw data are not available, the filtered data approach and its corresponding time window have been used to derive the photospheric model upper limits on the fluence for these two gamma ray bursts.

The MC signal simulations and the analysis optimisation are performed independently for each burst, while the background is estimated though data. The signal and background angular resolution are derived in order to compute pseudo-experiments, relying on an extended maximum likelihood ratio test statistic. This procedure is repeated requiring different track quality parameters: the set of cuts that maximize the model discovery potential is chosen. 


\section{Results}

No neutrino events were found spatially and temporally in coincidence with any of the four bright GRBs presented, so $90 \%$ C.L limits on the neutrino fluence have been computed for the internal shock model and the photospheric model (Figure 1).
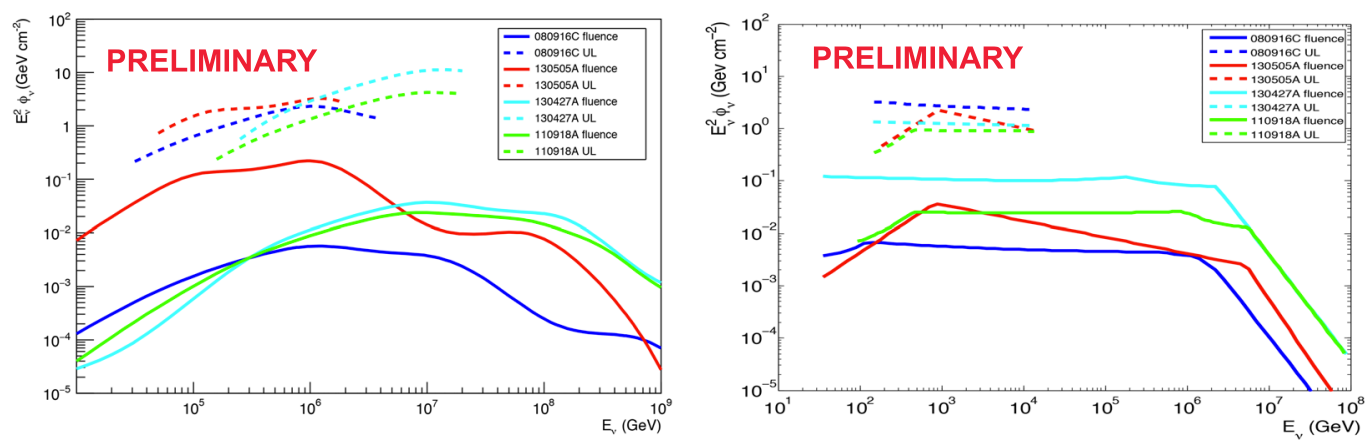

Figure 1. Expected $v_{\mu}+\bar{v}_{\mu}$ fluence (solid line) and ANTARES 90\% C.L. upper limit (dashed line) on the selected GRBs, in the energy band where $90 \%$ of the signal is expected to be detected by ANTARES according to internal shock model (left) and photospheric model (right).

\section{Constraints on GRB parameters}

The $90 \%$ C.L limits on the neutrino fluence allows setting constraints on the free parameters that significantly impact the neutrino flux such as the baryonic loading factor $f_{p}$ and the bulk Lorentz factor $\Gamma$. In Figure 2 the best $90 \%$ and 50\% C.L exclusion limits in the $\Gamma-f_{p}$ plane for both the internal shock model and the photospheric scenario are shown: they are respectively on GRB130505A and on GRB130427A. The results are obtained assuming that $f_{p} \in[1-200]$ and $\Gamma \in[10-900]$ and that the two parameters are not correlated.

The constraints on GRB130505A start to significantly challenge the internal shock scenario up to $\Gamma \sim 200$, while constraints on GRB130427A according to the photospheric picture can rule out a high baryonic content $\left(f_{p}<100\right)$ in its jet.

\section{Conclusions}

The analysis of the four most promising gamma ray bursts did not yield a measurement of a neutrino flux in correspondence of the GRB events, but it allows setting an upper limit on the neutrino flux from the gamma ray burst for two different emission models (internal shock and photospheric). Two dedicated analysis methodology have been developed in order to maximize the detector sensitivity for each model and both analysis were optimized in order to provide the highest model discovery potential for each burst. This measurement allows setting constraints on the parameters that affect the neutrino yield: the bulk Lorentz factor of the jet $\Gamma$ and the baryon loading $f_{p}$. A similar analysis has been performed by the IceCube collaboration [10] excluding small bulk lorentz boost factor and large baryonic loading. However the photospheric limits shown in Figure 1 (right) constrain emission at substantially lower energies than the IceCube results. 

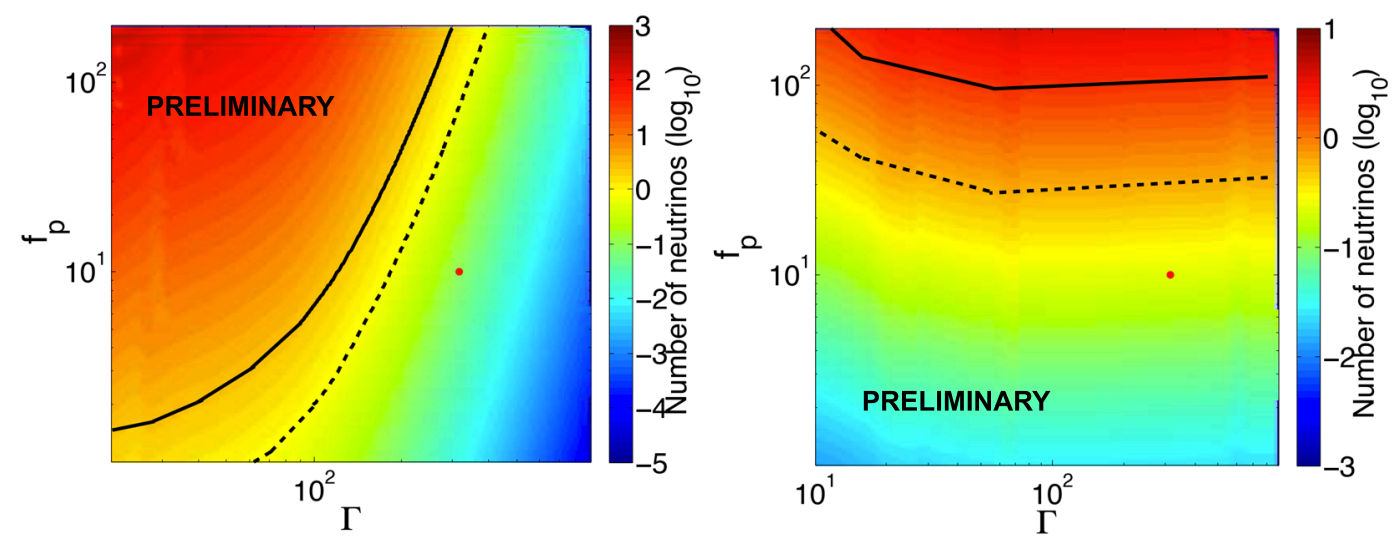

Figure 2. Constraints on the $\Gamma-f_{p}$ plane. The solid (dashed) black line corresponds to the exclusion limits at $90(50) \%$ C.L. The red dot shows the benchmark value $f_{p}=10$ and $\Gamma=316$. Internal shock scenario for GRB130505A (left) and photospheric scenario for GRB130427A (right).

The future KM3NeT-ARCA telescope will be the ideal instrument to constrain the neutrino flux from GRBs. It will be a kilometre scale detector composed of a two building blocks of 115 detection strings each. The detection of neutrinos from gamma ray bursts will provide important information of the process of emission of GRB, in particular it will give strong indications of the hadronic interactions occurring in the jets of the gamma ray bursts and it will help to distinguish between the different GRB model scenarios.

\section{References}

[1] T. Piran, Rev. Mod. Phys. 76, 1143 (2004), astro-ph/0405503

[2] P. Meszaros, Rept. Prog. Phys. 69, 2259 (2006), astro-ph/0605208

[3] P. Kumar, B. Zhang, Phys. Rept. 561, 1 (2014), 1410.0679

[4] S. Adrian-Martinez et al. (ANTARES), JCAP 1303, 006 (2013), 1302.6750

[5] S. Adrian-Martinez et al. (ANTARES), Astron. Astrophys. 559, A9 (2013), 1307.0304

[6] M.G. Aartsen et al. (IceCube), Astrophys. J. 805, L5 (2015), 1412 . 6510

[7] M.G. Aartsen et al. (IceCube), Astrophys. J. 824, 115 (2016), 1601.06484

[8] S. Hummer, M. Ruger, F. Spanier, W. Winter, Astrophys. J. 721, 630 (2010), 1002 . 1310

[9] S. Celli et al. (ANTARES), Proceeding of RICAP16, EPJ Web Conf. (2016)

[10] M.G. Aartsen et al. (IceCube), Astrophys. J. 824, 115 (2016), 1601.06484 